\title{
Redes para el Desarrollo: las instituciones educativas desde una perspectiva comunitaria
}

Mag. Andrea Tejera*

\section{Abstract}

El objetivo de este artículo es analizar a la organización educativa desde el enfoque de redes, subrayando los aspectos de interacción hacia lo interno y lo externo, en lo que respecta a todos los lazos comunitarios y desde una perspectiva de desarrollo local.

Los invitamos a pensar la institución educativa en su contexto particular, donde comparte un territorio con determinadas características, con otros grupos formales e informales con los que interactúa en diferente medida.

Al pensar de una manera diferente la propia organización interna de la institución educativa, más allá de la estructura formal, también vamos a encontrar un sinnúmero de relaciones que escapan al organigrama formal o explícito.

\section{Introducción}

Estamos acostumbrados a mirar y a pensar nuestras instituciones educativas "puertas adentro", es así que la mayoría de las veces analizamos a la organización desde la dimensión pedagógica, curricular y administrativo financiera quedando la dimensión comunitaria como la última y más desatendida.

Tendemos a pensar, sobre todo refiriéndonos al sistema educativo público, que el contexto es una situación azarosa, cuando en realidad el contexto debería ser la razón de ser de cada organización educativa: atender a determinada población con un conjunto de herramientas que le posibiliten el logro de aprendizajes válidos.

Hoy en día estos análisis se hacen cada vez más seguido, porque son las propias poblaciones las que interpelan nuestras herramientas y procedimientos y nos exigen una mayor creatividad.

Partimos de la consideración del liceo como una organización y como nuestra unidad de análisis y de gestión, entrecruzada por los valores -lo institucional- y por el sistema.

Sin lugar a dudas la escuela está atravesada por la red social y nocional: creencias, prejuicios y expectativas sobre roles. "La escuela es un entramado de relaciones sociales materiales que organizan la experiencia cotidiana y personal del alumno/a con la misma fuerza o más que las relaciones de producción puedan organizar las del obrero en el taller o las del pequeño productor en el mercado. ¿Por qué entonces, continuar mirando el espacio escolar como si en él no hubiera otra cosa en lo que fijarse que las ideas que se transmiten?"1. 


\section{La Institución educativa como unidad de análisis}

En la actuación de las personas en la vida organizacional no sólo influye la estructura de la organización y las estrategias que desarrollan los actores ${ }^{2}$, sino que entran a jugar factores como la formación, la historia familiar, y el contexto social. Por lo cual el uso de la libertad se ve condicionado por una serie de elementos que son particulares y situacionales -grupales y personales-.

Las organizaciones son construcciones sociales, llevadas adelante multidisciplinariamente por un sinnúmero de racionalidades subyacentes, desde las cuales la noción de organización es diferente. ${ }^{3}$

Schvarstein señala que las organizaciones en un tiempo y en un lugar materializan el orden social que establecen las instituciones. Define a las instituciones como aquellos cuerpos normativos jurídico-culturales compuestos de ideas, valores, creencias y leyes que determinan las formas de intercambio social.

En este sentido, lo instituido se comprende como aquello que está establecido, el conjunto de normas y valores dominantes, así como el sistema de roles que constituye el sostén de todo orden social. Para entender la dinámica del cambio social, es necesario reconocer la presencia de una fuerza instituyente, constituida como protesta y como negación de lo instituido.

A partir de estos planteos resulta fundamental, entonces, el abordaje de la organización desde su diversidad; las distintas historias, mitos, tradiciones, en definitiva, tener en cuenta los diferentes contextos sociales, culturales y económicos, en que están insertas y se desarrollan y las realidades de quienes las integran.

El que cada organización pueda tener en cuenta lo particular de su funcionamiento potencia su acción con relación al cumplimiento de sus objetivos. Por lo general lo que vemos es que las organizaciones pretenden ser homogéneas por medio de normas y reglamentos, que no tienen en cuenta las situaciones particulares, sino que regulan "de la forma más pareja posible" su funcionamiento; esto puede traer como consecuencia una pérdida de la eficacia y una descontextualización de las mismas.

Muchas veces se dice "la escuela debe abrirse a la comunidad": si no está en la comunidad ¿dónde es que se encuentra? Esto es un falso dilema y el hecho de concebir a las organizaciones desde sus redes de interacciones nos ayuda a desdibujar fronteras y a visualizarlas en la comunidad ${ }^{4}$.

Como señala Dabas, un mismo territorio está cruzado simultáneamente por diversas instituciones con sus organizaciones respectivas. Se considera que este territorio está también atravesado por la población, que plantea sus necesidades e intereses.

Como primer aspecto a recuperar es importante pensar que los cambios no provienen solamente de la macroestructura. Aun más, que los propuestos desde el sistema son imposibles de llevar a cabo sin la intervención activa y responsable de todos los agentes sociales involucrados.

Como señala Schvarstein aquellas organizaciones que construyen su identidad se constituyen en organizaciones-sujeto, por oposición al concepto de organización-objeto que caracteriza a aquellas cuyo orden está determinado externamente. 


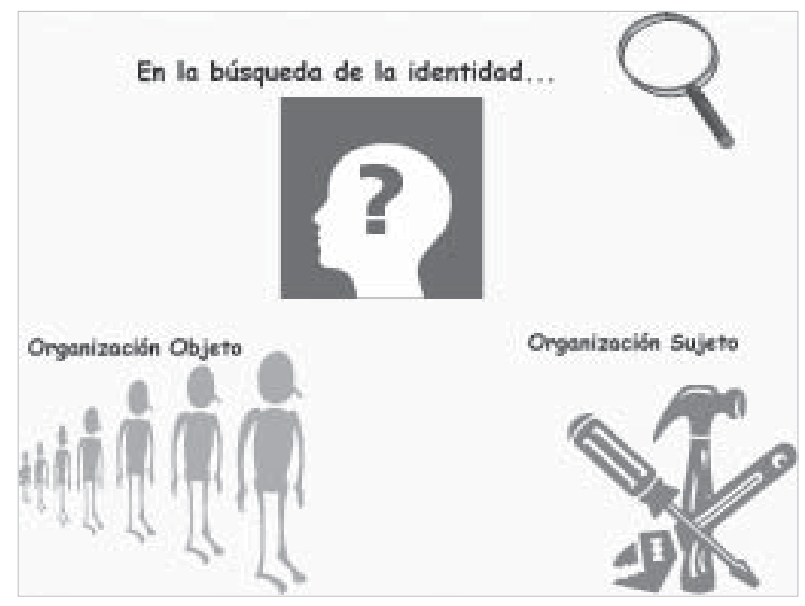

Lo que permite caracterizar a un grupo como grupo-sujeto es su capacidad instituyente, la posibilidad de modificar y ser modificado por la organización a la que pertenece. Construye su propia trama argumental y es capaz de sostenerla a lo largo del tiempo; constituye una modalidad discursiva con sus integrantes, de modo que cada uno de ellos es una parte a su vez portadora y reproductora del todo, favoreciendo el pasaje de la afiliación a la pertenencia. Las organizaciones-objeto desarrollan una trama argumental que fue escrita por otro, son enunciatarias del discurso de otro.

\section{Organización y contexto}

En esta trama consideramos a la institución educativa como actor institucional, en el sentido de organización que ocupa un lugar en el territorio y en la cual se toman decisiones que pueden afectar en mayor o menor grado a la comunidad local. Definiendo a la comunidad como los "subsistemas sociales de base, social, económica y ecológicamente heterogéneos, que comparten una parte relevante de la vida cotidiana y de las condiciones colectivas de reproducción de sus miembros"5.

La comunidad es el contexto del cual cada institución educativa forma parte, entendido éste como la sociedad humana a cuyo desarrollo la educación quiere contribuir, o sea el conjunto de circunstancias en las que se inserta la institución educativa; por lo tanto no sólo consideramos al contexto una condición más o menos favorable para la educación, sino el objeto mismo de la intervención educativa.

Es decir, es esa población concreta en la cual la organización se encuentra inserta, la que es objeto de su intervención y todas sus estrategias de enseñanza y aprendizaje deberían estar dirigidas y asentadas en sus características específicas.

Entonces, el contexto no es sólo lo que rodea a la institución sino que ella se encuentra inserta en él y es fundamental que logre un conocimiento profundo de sus características, para que los recursos de la organización puedan ser orientados de acuerdo a las necesidades particulares de cada población y así puedan lograrse procesos de enseñanza y de aprendizaje de calidad. 
Asimismo cuando nos referimos al territorio lo caracterizamos como un sistema dinámico de elementos físico-naturales, estéticos, culturales, psico-sociales y económicos que interaccionan con los hombres y con la comunidad en que viven.

Cada localidad es el resultado de una historia que se ha ido construyendo, con sus características sociales, políticas, económicas y culturales. En qué medida la organización educativa se permite conocer y se da a conocer en ese entorno es una pregunta que probablemente tenga diversas respuestas en función del territorio sobre el cual descansemos nuestra mirada.

Investigaciones y estudios realizados en torno a la reforma educativa llevada adelante en nuestro país a partir del año 1996 marcan la importancia del factor territorial en la estratificación de los aprendizajes y manifiestan los problemas de inequidad y de deterioro de la calidad de los mismos, identificándose causas más de fondo como "el paulatino agotamiento de la capacidad real del sistema educativo de responder exitosamente a las expectativas y necesidades, que surgen como crecientemente heterogéneas y segmentadas y asimismo, el desdibujamiento de la función de integración social, factor éste fundamental en la mitigación de las situaciones de pobreza y marginalidad"'.

En una investigación realizada en el marco del Programa de Mejoramiento de la Calidad de la Educación Primaria (MECAEP) se incluye la variable "área geográfica" planteándose que "hace referencia a las diferencias existentes entre Montevideo y su área metropolitana por un lado, y el interior del país propiamente dichas por el otro. Según podrá apreciarse más adelante, se trata de una variable que está correlacionada con los resultados ajustados y que parece estar indicando la existencia de diferentes niveles de integración social y de compromiso con la escuela en los diversos contextos demográficos. Las zonas más urbanizadas parecen caracterizarse por menores grados de integración social y de compromiso con la escuela, lo que determina menores niveles de aprendizaje. Dicho en otras palabras, el significado del carácter "desfavorable" del contexto sociocultural de la escuela es diferente en el interior del país que en Montevideo y su área metropolitana, resultando "más desfavorable" en esta última", $\operatorname{ANEP}(1999: 51)$.

Como bien señala Schvarstein, es la consideración del contexto la que debe orientar la acción de los integrantes, y hay que dotar a la organización de la necesaria flexibilidad para que se adapte a las demandas del medio externo. "... la capacidad de sostener las propias coherencias internas en el marco de un acople estructural con el contexto es lo que distingue a las organizaciones que más perduran en el tiempo. Una historia de interacciones a través de las cuales la organización realiza y afirma su propia identidad es lo deseable", Schvarstein (1991:15).

Teniendo presente la interrelación dinámica entre organización y contexto podemos empezar a reflexionar acerca de la contribución de las organizaciones al desarrollo local.

Entendemos que el desarrollo local se produce cuando existe capacidad de negociación y de juego entre actores, buscando una articulación de intereses que desemboque en beneficios para la sociedad del lugar? 
Podemos tomar la definición que nos brinda Arocena sobre actor local: "Una forma de definir un actor es relacionándolo con la escena social en la que desarrolla su acción. En este caso nos estamos refiriendo a individuos, grupos o instituciones cuyo sistema de acción coincide con los límites de la sociedad local. (...) la noción de actor local se liga a la noción de desarrollo. Ello supone incluir en la definición no sólo la escena social en que se desarrolla la acción, sino también el aporte que la acción realiza al desarrollo local", Arocena (1995: 26).

El espacio local como ámbito construido y vivido en su dinámica de evolución, tomando la noción de espacio en un sentido multidimensional: físico, social, cultural y simbólico. En tal sentido existe una gran variedad de espacios locales ya sea por la especificidad geográfica o por los actores que en ellos desarrollan sus vidas, las actividades de producción, los aspectos sociales y culturales. Estos espacios también tienen múltiples articulaciones con el global, nacional y regional.

Desde este punto de vista, el entorno no se concibe como una entidad global. Brofenbrenner, investigador ruso-americano, distingue en el mismo cuatro estructuras, considerando que cada una de ellas está contenida en la siguiente ${ }^{8}$.

El ambiente se extiende así más allá de la situación inmediata que afecta a la persona en desarrollo y comprende a la de persona, el contexto de su familia, la comunidad, la sociedad con instituciones establecidas (salud, religiosas, educativas y jurídicas) y la cultura.

Señala que cada persona se halla en una realidad que consiste en un número de sistemas de diferentes niveles, citando el ejemplo del sistema de que forma parte un niño, podemos describir:

Microsistema- familia o la persona cuidadora.

Mesosistema- escuela, centro recreativo, el grupo de amigos; nuevos contactos a medida que el niño crece.

Exosistema- sistemas de los que el niño no participa directamente, pero que lo afectan igual, por ejemplo otro trabajo de la cuidadora.

Macrosistema- sistemas de nivel abarcativo, en los cuales todos dependen unos de otros y cooperan entre sí, por ejemplo la religión y las leyes.

\section{La cultura institucional}

Así como pensamos que todos los liceos o escuelas son iguales, hacemos lo mismo respecto a las personas que en ellos trabajan. Hablamos del colectivo docente y administrativo, como si fueran homogéneos.

Sin embargo, cada organización posee rasgos de identidad y señas particulares que le son propias, cultiva un estilo particular, que se construye en un complejo entramado, en el cual los objetivos pautados por la organización van siendo moldeados por los actores, en lo manifiesto y en lo menos visible.

Por lo tanto los actores tanto individuales como grupales poseen recursos personales y 
culturales que les permiten moverse de una forma particular en las organizaciones.

En este sentido resulta pertinente incorporar el concepto de cultura institucional: constituida por valores, creencias, representaciones, expectativas, tradiciones y simbologías propias, roles, có

"La cultura institucional se ofrece como un entramado de sostén y un organizador de la interacción y el trabajo de grupos y sujetos y también como apoyatura psíquica de aspectos sustantivos de la identidad individual", Fernández, (1999).

nes

"La cultura institucional es aquella cualidad relativamente estable que resulta de las políticas que afectan a esa institución y de las prácticas de los miembros de un establecimiento. Es el modo en que ambas son percibidas por estos últimos, dando un marco de referencia para la comprensión de las situaciones cotidianas y actividades de todos aquellos que actúan en ella" (Frigerio, G. y ot.1992. Las instituciones educativas Caras y Ceca. Buenos Aires:Troquel Educación).

\section{Redes para el desarrollo}

El concepto de redes tiene múltiples sentidos: método de análisis social, modalidad de pensar las prácticas sociales y propuesta organizacional.

Vamos a definir las redes como sistemas abiertos -no son grupos cerrados- mediante los cuales se produce un intercambio dinámico -basado en la reciprocidad-tanto entre sus propios integrantes como con integrantes de otros grupos y organizaciones, posibilitando así la potencialidad de los recursos que poseen ${ }^{9}$.

"Las redes son estructuras abiertas, capaces de expandirse sin límites, integrando nuevos nodos mientras puedan comunicarse entre sí, es decir siempre que compartan los mismos códigos de comunicación (por ejemplo valores o metas de actuación). Una estructura social que se base en las redes es un sistema muy dinámico y abierto, susceptible de innovarse sin amenazar su equilibrio"10 .

El autor pone énfasis en las redes como vehículos por los cuales circula la información: redes entre empresas; dentro de las empresas; personales e informáticas.

Partimos de la consideración de situar a los seres humanos no como individuos aislados sino como partes integrantes de diversas redes. Todos en algún momento hemos sentido el aislamiento tanto dentro de un grupo como en afuera; la preocupación por la dinámica interna de las instituciones, la cultura individualista y competitiva muchas veces no nos dejan ver que hay quienes comparten no sólo nuestros problemas, sino también nuestra manera de pensar y vivir. También las soluciones que nosotros elaboramos les podrían servir a otros.

Las personas son parte de múltiples redes de interacciones: familiares, de amistad, laborales, recreativas, políticas, culturales e informativas. Las redes son pre-existentes pero apoyándonos 
en ellas podemos potenciar lo que hay y lo que queremos.

Estamos poniendo en evidencia dos características principales de la red:

1. sistema abierto: admite ingreso y egreso.

2. multicentralidad de la red: modifica el paradigma de la pirámide, la red apela permanentemente a la reciprocidad.

Esto nos habla del poder distribuido, las jerarquías remiten a las relaciones piramidales unidireccionales, las redes nos remiten a la multidireccionalidad -nodos-.

Cada miembro se enriquece a través de las múltiples relaciones que cada uno de los otros desarrolla, la riqueza de las redes está en la multiplicidad de singularidades.

Desde este punto de vista, no es necesario discriminar las relaciones dentro y fuera de las organizaciones educativas, sino que la frontera entre organización y entorno se desdibuja y podemos focalizar en la interacción en sí.

Se plantea la necesidad de concebir la realidad en términos de relaciones, de pautas que conectan. Desde esta perspectiva se evidencia que las redes han existido desde siempre, dentro de una realidad dinámica, cambiante ${ }^{11}$.

Desde la perspectiva de red, por ejemplo en lo familiar otras personas, además de los padres, pueden desarrollar una relación de sostén del niño, la cual puede volverse decisiva. Para el caso del docente y de la organización liceal, podemos pensar que otras personas pueden desarrollar una función de enseñanza y otros espacios pueden ser educativos.

Esta concepción tiene impactos en todos los aspectos de la vida institucional. Hoy en día se hace cada vez más necesario partir del reconocimiento de la heterogeneidad de las instituciones, de los entornos y de las personas, con lo cual ya no podemos pensar en tener respuestas exitosas frente a mecanismos de convocatoria y participación masivos -homogéneos-, sino que tenemos que pensar en las particularidades, en las habilidades y saberes específicos de cada uno de los actores de la multiplicidad.

\section{Redes internas a la institución}

Podemos focalizar nuestra mirada hacia lo interno de la organización y teniendo en cuenta los vínculos que establecen las personas con otros, vamos a encontrar en la maraña de relaciones que se establecen en lo interno de la institución, lazos más concentrados, que podríamos traducir en pequeños núcleos de relaciones.

Desde este punto de vista, ya no estaríamos pensando la institución como un conjunto de individuos, sino como un conjunto de relaciones integradas y por diversos núcleos que comparten afinidades, compromisos y saberes.

Esto se puede evidenciar muchas veces a través del discurso, cuando hablamos de nosotros y ellos, allí estamos y nos estamos posicionando en relación a un conjunto de personas y nos estamos excluyendo respecto de otros, y a través de las relaciones recurrentes en un grupo. (por ejemplo, los del mismo turno, los de la misma asignatura o área, docentes jóvenes, docentes con más experiencias, docentes con mayor antigüedad en la institución, docentes de la localidad 
y docentes que viajan).

En el centro educativo existen un conjunto de relaciones formales e informales que componen un complejo entramado de redes comunicativas: superficiales y centrales; verbales y gestuales; fácticas y omisivas; con ejes bipolares y multipolares.

El organigrama ${ }^{12}$ puede ser definido como la disposición racional, consciente e institucionalizada de la división del trabajo. La imagen que tenemos del organigrama se corresponde con la concepción weberiana de organización burocrática, según la cual la división del trabajo tiene que llevarse a cabo mediante divisiones funcionales no solapadas, con una jerarquía de coordinación y control y con procedimientos y reglas de actuación que garantizan relaciones formalizadas e impersonales entre sus miembros.

Como bien señala Molina, los diferentes niveles de la organización burocrática se justifican no sólo por sus funciones de control (en el organigrama hay funciones especiales para la supervisión) sino por su función de coordinación de actividades, necesaria por la especialización en unidades para aumentar su productividad y eficacia (Figura 1).

Figura 1. Estructura del organigrar Escriba aquí el nombre Escriba aquí el cargo

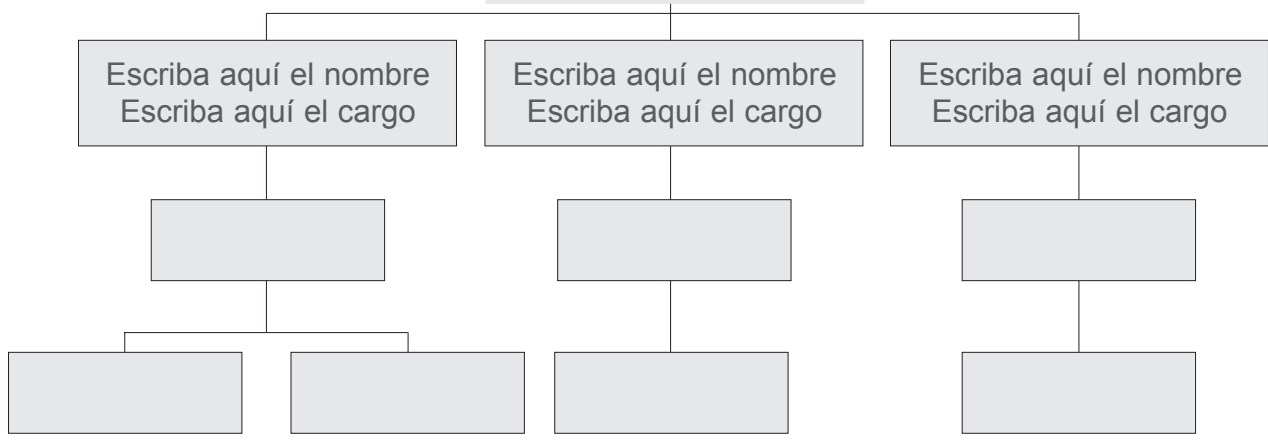

Hay autores que hablan de la red como el organigrama informal, en el que confluyen las relaciones de trabajo, las relaciones de amistad y las relaciones de consejo.

Esto nos habla del poder distribuido, las jerarquías remiten a las relaciones piramidales unidireccionales, las redes nos remiten a la multidireccionalidad -nodos-. El concepto de heterarquía se refiere a la posibilidad de coexistencia de "jerarquías" distintas en el funcionamiento de un sistema determinado. No implica la negación de la jerarquía sino la 
coexistencia y el reconocimiento de una variedad de las mismas.

Figura 2 - La estructura

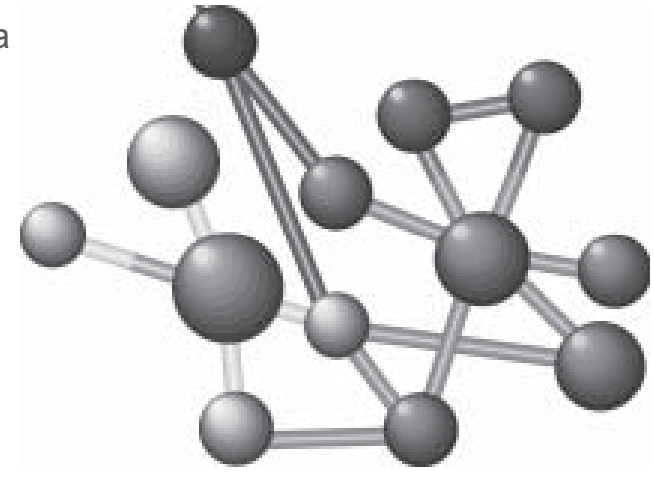

En el interior de estas redes se establecen relaciones más fluidas que permiten la circulación de la información y de los recursos, por lo tanto a cuantas más redes pertenezca una persona o cuantas más tenga una institución, más rica en recursos, en información y en procesos comunicacionales será ${ }^{13}$.

\section{Lazos fuertes y débiles}

Es importante también tener en cuenta el tipo de lazos que se establecen en estas redes ya que los lazos demasiado fuertes -que tienden a producir pequeños grupos muy unidos-, generalmente llevan al aislamiento, en cambio los lazos más débiles forman puentes entre grupos, ampliando el número y la variedad de los contactos, siendo más tolerantes a la diversidad y más proclives a la innovación ${ }^{14}$.

En el primer caso se trata de círculos cerrados, cuya principal carencia son los lazos que los podrían conectar a otras dimensiones de la vida institucional. Podría pensarse que si los proyectos alcanzan un pequeño grupo y tienen problemas para ampliar la participación, se debe a que no rompen los bordes de círculos institucionales sumamente cerrados, de ahí en parte, las referencias que siempre se dan con relación a las grandes dificultades de integración.

\section{Redes externas a la institución}

El fortalecimiento de la red interna es el primer paso para la ampliación de las fronteras. La profundización de los vínculos lleva a reconocer la diversidad de los saberes, de las habilidades, de los contextos de inserción y de las posibilidades que cada uno de ellos tiene.

Implica empezar a ver los puntos de conexión entre las diferentes políticas sectoriales y sus instituciones porque son los mismos pobladores; hacer redes requiere comenzar a mirarnos entre todos y observar el entorno.

Como bien señala Dabas, un mismo territorio está cruzado simultáneamente por diversas instituciones con sus organizaciones respectivas. Desde la perspectiva de redes se considera que este territorio está también atravesado por la población, que plantea sus necesidades e intereses.

De la menor o mayor cantidad de esas "redes de relaciones" ha de depender la debilidad o la fortaleza de cada organización y, por lo tanto, de sus integrantes, lo cual, a la vez, condiciona 
la modalidad en que éstos se integran a la sociedad de la que forman parte.

\section{Pensando en la intervención}

Desde el punto de vista del análisis o la intervención, hay determinadas características que pueden tenerse en cuenta; por ejemplo, pensando en redes personales es posible analizar:

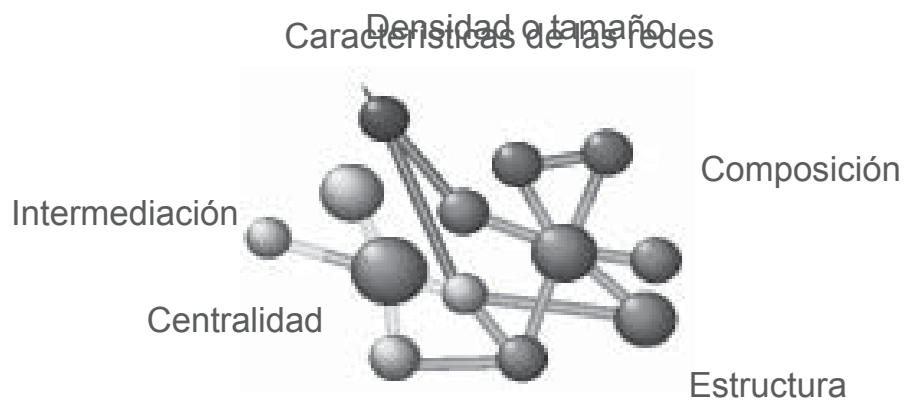

- tamaño de la red o densidad (número de relaciones presentes sobre el número de relaciones posibles)

- composición de la red, "tipos de relaciones" (pedir al informante que represente varias categorías de personas -familiares, amigos, vecinos, compañeros de trabajo-)

- estructura de la red: lineal y piramidal.

- centralidad, ver quién mantiene más relaciones con los otros o la facilidad con que una persona se conecta con otras familias. Es el estudio de la posición de cada actor en la red.

- Intermediación, número de veces que un actor cae en el camino más corto entre otros dos actores.

En una investigación de estudio de caso realizada en el año 2000 en un liceo de la periferia de Montevideo y ante la pregunta de cuáles son las características de los actores institucionales que facilitan las relaciones con la comunidad, de acuerdo con lo que hemos analizado, se informaba:

Las relaciones con la comunidad en los docentes, se ven facilitadas por la mayor antigüedad, la concentración de horas y la residencia local para el caso del conocimiento de los vínculos del liceo con otras instituciones. En el caso de la participación en actividades de la comunidad, la concentración de horas y la residencia local son factores determinantes.

Para el conocimiento de las relaciones que mantiene el liceo con otras instituciones, existiría cierta paridad en cuanto al peso de ser antiguo, concentrado o residente local.

Respecto a la "participación", sin lugar a dudas para poder participar de una actividad comunitaria se necesita estar en la institución -tener concentración horaria- o por lo menos vivir en la zona. En eso influyen aspectos que tienen que ver con el compromiso que uno puede establecer por sentirse perteneciente a la localidad y a la institución y con aspectos 
prácticos, en cuanto a posibilidad real de participar.

Los actores que a partir del análisis de la información relevada resultan como grandes facilitadores de las "relaciones con la comunidad" son, sin lugar a dudas, los identificados como personal de docencia indirecta: adscriptos, profesor orientador pedagógico, coordinadora de la experiencia de innovación, asistente social, directora y secretaria administrativa. El cúmulo de relaciones en cuanto a conocimiento y participación que establecen, es ampliamente superior al de los docentes.

Las coordinaciones, como espacio de encuentro y trabajo en equipo, parecen ser otro factor que influye en el logro de una red intrainstitucional, habilitante a la hora de vincularse y participar en representación.

La actitud del cuerpo docente, respecto al relacionamiento con la comunidad, parece ser de apertura, se percibe como una necesidad, sobre todo en el personal de docencia indirecta.

Estos actores se constituyen como referentes importantes de la institución, al parecer tanto con respecto a lo interno como hacia lo externo de la misma, hacen latir al liceo.

En esta investigación se pone en evidencia, por un lado, que para que se enriquezca la trama de interconexiones son necesarios cambios en al ámbito del sistema educativo que potencien y faciliten una mayor autonomía de las organizaciones educativas y cambios en cuanto a los procedimientos de elección de horas de los docentes y la elección de cargos de los directores, en los que se privilegien fundamentalmente aspectos de su residencia, pero también con relación a mecanismos que favorezcan la concentración horaria.

Por otro lado se pone de manifiesto el peso del director en las vinculaciones con el entorno, ya que en el estudio se visualiza su liderazgo en cuanto actor local, pero sería bueno investigar qué ocurre cuando el director no lo es.

Deberían establecerse conexiones que favorecieran los procesos de aprendizaje y colaboraran en la superación del aislamiento que a veces manifiestan sentir las instituciones educativas.

En definitiva, esta mirada sobre las redes nos permite visualizar desde una perspectiva integral los problemas compartidos por una comunidad, y la necesaria complementariedad de las acciones implementadas para solucionarlos. Desde esta perspectiva todos los actores tienen implicancia y la historia cuenta.

El trabajo en proyecto ofrece oportunidades para fortalecer la red interna. El reconocimiento de esta dimensión de la realidad exige una transformación en nuestros mecanismos de participación y de convocatoria, requiere un "ir hacia la gente" y no un "esperar que vengan", acercarnos a esos núcleos para saber qué opinión tienen, pensarlos en función de su saber específico. No hay que olvidar que una estrategia de participación o de convocatoria es una forma de acumular relaciones para un logro determinado.

Por lo tanto se hace necesaria una resignificación de los procedimientos y las reglas, desritualizarlas y adjudicarles un nuevo sentido en función de los objetivos organizacionales. Para ello, antes del diseño y realización de las tareas, resulta necesario tener en claro, de la forma más colectiva posible, los beneficios que nos pueden proporcionar. 


\section{Bibliografía y notas}

${ }^{1}$ Gimeno, J. y Pérez, A. 1995. Comprender y transformar la enseñanza. Madrid: Ediciones Morata.

${ }^{2}$ Crozier, M. Friedberg, E. 1990. El actor y el sistema. Las restricciones de la acción colectiva. México: Alianza Editorial Mexicana.

${ }^{3}$ Schvarstein, L. 1991. Psicología Social de las Organizaciones. Buenos Aires: Editorial Paidós.

4,9,11 y 13 Dabas, E. 1998. Redes sociales, familias y escuela. Buenos Aires: Editorial Paidós.

${ }^{5}$ Coraggio, J. 1995. Desarrollo humano, economía popular y educación. Buenos Aires: Aique.

${ }^{6}$ ANEP. 1998. La Reforma Educativa en Uruguay. Presentación por el Director Nacional de Educación Pública, Profesor Germán W. Rama. Montevideo.

${ }^{7}$ Arocena, J. 1995. El desarrollo local un desafío contemporáneo. Caracas: Ediciones Nueva Sociedad.

${ }^{8}$ Brofenbrenner, U. 1983. Ecología del Desarrollo Humano. Buenos Aires: Editorial Paidós.

10 Castells, M. 1997. La era de la información. Economía sociedad y cultura. La sociedad red, Vol. 1. Madrid: Alianza Editorial.

12 Molina, J. L. 2001. El organigrama informal en las organizaciones. División Antropológica Social, Universidad Autónoma de Barcelona.

${ }^{14}$ Espinoza, V. 1995. Redes Sociales y Superación de la Pobreza. Revista de Trabajo Social, № 66. Escuela de Trabajo Social, Pontificia Universidad Católica de Chile.

*Magister en Desarrollo Regional y Local, Universidad Católica del Uruguay. Licenciada en Psicología, Universidad de la República. Directora, Programa de Apertura y Consolidación, Plan CAIF-INAME. Docente e investigadora, Consejo de Educación Secundaria. 